\title{
Multi-Level Dataflow-Driven Macro Placement guided by RTL Structure and Analytical Methods
}

\author{
Alex Vidal-Obiols, Jordi Cortadella, Jordi Petit, Marc Galceran-Oms, Ferran Martorell
}

\begin{abstract}
When RTL designers define the hierarchy of a system, they exploit their knowledge about the conceptual abstractions devised during the design and the functional interactions between the logical components. This valuable information is often lost during physical synthesis. This paper proposes HiDaP, a novel multi-level algorithm that uses RTL information and analytical methods for the macro placement problem of modern designs dominated by multi-cycle connection pipelines. By taking advantage of the hierarchy tree, the netlist is divided into blocks containing macros and standard cells, and their dataflow affinity is inferred considering the register latency and flow width of their interaction. The layout is represented using slicing structures and generated with a top-down algorithm capable of handling blocks with both hard and soft components. An adaptive multi-objective cost function is used to simultaneously minimize wirelength, timing, overlap and distance to preferred locations, which can be user-defined or generated by analytic methods (spectral and force-directed). These techniques have been applied to a set of large industrial circuits and compared against state-of-theart commercial and academic placers, and also to handcrafted floorplans generated by expert back-end engineers. The proposed approach outperforms previous algorithmic methods and can produce solutions with better wirelength and timing than the best handcrafted floorplans. Post-routing layouts are almost brought to timing closure and DRC cleanness with minimal engineer modification, showing that the generated floorplans provide an excellent starting point for the physical design flow and contribute to reduce turn-around time significantly.
\end{abstract}

\section{INTRODUCTION}

Current industrial EDA floorplanning solutions are fast but often do not produce good enough macro placements. Additional iterations by physical designers, which take significant effort, are needed. For circuits with more than 200 macros, it usually takes 2 to 4 weeks for the floorplan to reach the desired quality of results. As decisions taken at the floorplan stage have a critical impact on timing and power, considering structural properties during macro placement helps reduce design turn-around time.

A key challenge in advanced technology nodes is that connections between components inside a same block can take more than one cycle. This is considered during RTL design, when wires are pipelined, based on RTL designer expertise or previous versions of the design, to meet timing constraints. Awareness of RTL structure when deciding the location of macros is key to obtain floorplans which can close timing

*A. Vidal-Obiols, M. Galceran-Oms and F. Martorell are with Inphi Corporation. J. Cortadella and J. Petit are with the Department of Computer Science, Universitat Politècnica de Catalunya. This work has been partially supported by a grant from Inphi Corporation and funds from the Spanish Ministry for Economy and Competitiveness and the European Union (FEDER funds) under grant TIN2017-86727-C2-1-R, and the Generalitat de Catalunya (2017 SGR 786).

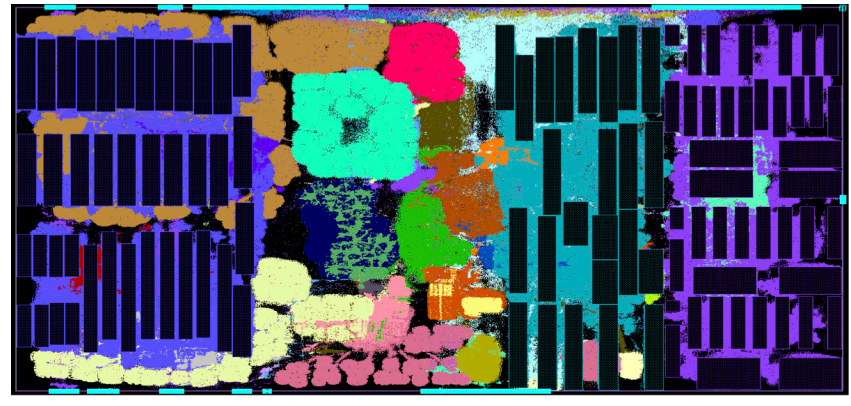

(a) $c 7$ automatic macro placement.

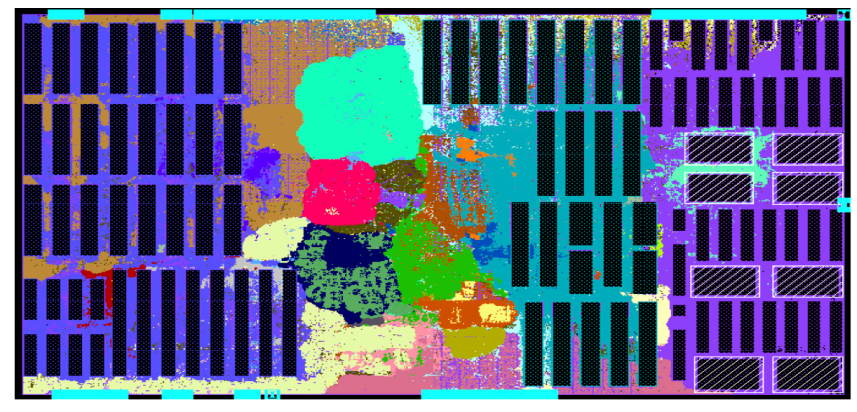

(b) $c 7$ after light manual refinement.

Fig. 1: HiDaP can generate close to sign-off-ready macro placements on challenging designs that our reference EDA tool does not manage to route.

without excessive congestion. The dataflow metric proposed in this work aims to capture the relation between cell groups, ports and macros by considering the number of bits and number of stages in the pipeline connections between them.

The dataflow analysis of a given design is achieved by exploiting RTL-stage information, such as hierarchy and arrays. Many algorithms devote high computational effort to infer structural properties that were known in previous stages of the design and have been destroyed or ignored. The hierarchical abstraction used by humans provides a logic vision of the relations between circuit components. Identifying array components (registers, ports...) is key to understanding the relations between macro components (and the standard cells) and providing floorplans that are more attuned to the structure of the system.

This paper proposes HiDaP (for Hierarchical Dataflow Placement), a modern macro placer to help finding better macro placements in an industrial physical design flow. Circuit partitions and their relations in terms of dataflow are derived by using readily-available RTL-stage information present in the netlist. An adaptive multi-objective cost function is used to reduce wirelength, timing, overlaps and distance to preferred 
locations, which can be provided by engineers or generated automatically by analytical methods. A macro placement obtained with HiDaP exhibiting good timing properties can be seen in Fig. 1a A back-end engineer brought the design to only 400 failing endpoints and 22 DRC violations in one round of light manual refinement (Fig. 1b). For this circuit, our reference industrial EDA tool generated a macro placement that could not be routed afterwards. This is the first time that macro placement algorithms have been compared against manually-crafted sign-off-ready macro placements, while providing industrial QoR metrics (including WNS/TNS) after place and route.

\section{Related work}

Some approaches to mixed-size placement such as RePlAce [10] (integrated in the open-source OpenROAD initiative [4]) perform global placement using analytic techniques. These techniques tend to first optimize the design and then remove overlaps, which sometimes leads to degrading the previous optimization or not reaching good layouts in terms of legalization. To prioritize legal macro placements and in order to enable the modular incorporation of HiDaP into an industrial flow (so that it is independent of the later std. cell placement stage done using industrial methods), our method advocates to perform macro placement prior to cell placement.

One of the main challenges of our approach is that by choosing to place macros first, even though placement prototyping may be used, standard cell locations are not known. A very prolific family of macro placers considers cell area implicitly by having macros close to circuit walls, which is the de facto chosen approach for some industrial floorplanning tools. The method was initially proposed by [8] and relied on packing macros at the corners of the circuit using MP-trees. It was extended by [9] for routability and blockage-awareness. In [11], three major drawbacks to the use of these previous approaches in modern flows (related to overlap avoidance, preplaced macros, and area utilization) were presented and $\mathrm{CP}$ trees were proposed, aiming also to optimize the shape and area of the region for standard cells. However, the approach lacked scalability. The most recent advances in this direction propose a multi-level framework for scalability [6], extensions to consider macro and cell interaction [7] and domain planning with macro placement [24].

In contrast with all these packing methods, our approach advocates for directly modeling cells during macro placement to avoid forcing them to the macro walls, where they might be far away from their corresponding standard cells. It is not clear how to model the wirelength between macros and allocate whitespace without considering standard cells, as having all macros in the middle could possibly generate good solutions in terms of wirelength but unacceptable for routability and timing closure. Although detailed placement of standard cells is out of the scope of this work, floorplanning standard cell groups is key to find satisfying macro placements. Whereas other works consider floorplanning two kinds of blocks, hard for macros with a fixed shape, and soft for standard cell groups with a flexible shape, e.g., using sequence-pairs [3] or slicing trees with shapes curves [34], our approach proposes to model blocks with characteristics of both.

The RTL information present in the netlist is often used to help obtain better results during physical design. One of the ICCAD 2012 CAD contest problems was on Design hierarchy aware routability-driven placement [31]. Hierarchy information is used to create pseudo-nets to model the wirelength between macros in $[8]$ and the deriving approaches. It is also used in [13] to obtain better routability, and to guide placement in [17] [16]. More recently, the approach has been used in particular for better component clustering [21] [22].

The natural way in industrial flows to tackle the complexity of working with macros and cells is to use hierarchical and multi-level approaches. Some proposals [12] [2] [14] divide the layout in subregions and solve their placements independently, but not explicitly exploiting the hierarchy information of the circuit. Multi-level techniques, which follow either a cluster-decluster or partition-merge scheme, have been employed in other contributions [5] [28], and has lately been used to overcome the scalability issues of CP-trees [6]. HiDaP combines RTL hierarchy information with multi-level optimization to reduce problem size in macro placement.

One of the key questions all macro placers try to answer is: What makes a macro placement good? Most macro placers aim to primarily reduce some abstraction of wirelength, and also optimize other objectives like reducing overlap and displacement from desired positions [8] [24], have continuous cell area [6], improve routability [9] [23] or increase regularity [23]. Some approaches minimize complex objective functions in which the weight of components is tuned via user-defined parameters. In order to avoid manual tuning of the optimization function, our work implements an adaptive scheme in the vein of the one proposed in [19] to automatically decide those values depending on the current instance execution.

The usual physical design flow when pipelined connections are present in the block is an iterative process in which the designer tries to work on a floorplan to close timing and, when not possible, the pipelining is modified at the RTL level until a valid floorplan is found. These issues were considered in the context of microarchitecture-aware floorplanning [25] or bus-aware floorplanning [27] [18], but their efforts were not centered on how to model these connections from a netlist. Another recent work also identifies the need to understand indirect connectivity between macros [22], but not the timing problems induced by register pipelines. Our work presents the first macro placement method that considers in-block multicycle connections and exploits array information to model dataflow with the aim of reducing both circuit wirelength and timing in modern macro placement.

\section{Our contribution}

This paper introduces HiDaP, a multi-level floorplanner to find macro placement and orientation by exploiting RTL structural information. The hierarhical process is illustrated in Fig. 2 by defining the location of the 16 macros of a design. As shown in Fig. 2a the first partition identifies three key components, two containing 8 macros each, and a third with 


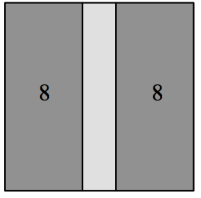

(a)

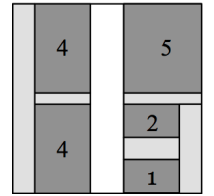

(b)

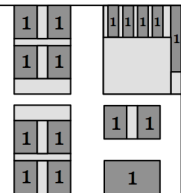

(c)

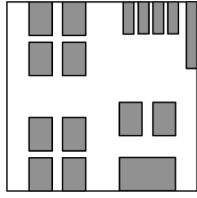

(d)
Fig. 2: Evolution of the multi-level block floorplan of a 16macro design. Dark gray blocks contain macros, count inside. Light blocks contain only standard cells.

cells connecting them. The process is repeated for the blocks at the left and right, and each is again partitioned into blocks with macros and cells and blocks with only cells (Fig. 2b). After a final recursive call (Fig. 2c), coordinates for each one of the 16 macros are fixed and space has been left for their related standard cells (Fig. 2d).

After examining the circuit hierarchy, our tool creates a partition into blocks (containing both macros and standard-cell groups) and analyzes circuit array information to estimate their dataflow affinity, based on component bit counts and pipeline connection stages. The layout of blocks is decided using slicing structures and simulated annealing, with a novel topdown layout generation algorithm designed to handle blocks with both hard and soft properties, not just hard as in most approaches derived from [33]. The approach considers the fixed die area to be a budget and distributes it among the blocks, implicitly dividing whitespace and avoiding overlap when possible.

The aim of our method is to reduce wirelength and timing without macro overlap, so that the circuit can reach timing closure without DRC violations with minimal manual intervention. A multi-objective cost function is introduced to model the problem of minimizing wirelength and timing (dataflow), distances to preferred locations and overlaps. These preferred macro locations can be introduced as additional input from the engineers or generated using analytical techniques (such as spectral and force-directed methods) to guide the search, reducing variability and increasing solution quality.

$\mathrm{HiDaP}$ has been tested on a suite of large industrial designs and compared against a state-of-the-art industrial floorplanner, a state-of-the-art academic placer (RePlAce [10]) and handcrafted macro placements. HiDaP improves on both floorplanning tools, and the obtained results are close to handcrafted designs (in particular with better timing results) with a fraction of the effort involved, providing a more promising start for the physical design iteration process.

Our key contributions include:

1) Multi-level macro placement based on recursive block floorplanning with hierarchy-aware declustering.

2) Multiple graph-based data structures to model the circuit and estimate dataflow affinity between blocks, considering register pipeline latencies and widths.

3) Block area modeling considering macros and standard cells, with a top-down area-budgeting strategy aiming to avoid overlaps.

4) Adaptive multi-objective algorithm to simultaneously reduce wirelength, timing, overlaps and distances to pre-

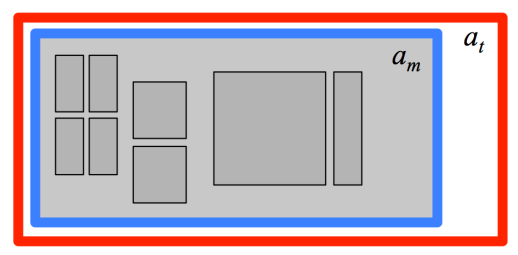

(a) Minimum and target areas.

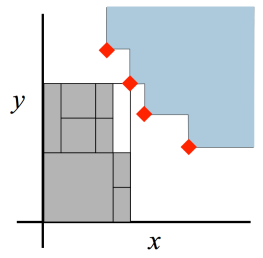

(b) Shape curve $(\Gamma)$.
Fig. 3: Area model for a block.

ferred macro locations without manual parameter tuning.

5) Simulated annealing search guided by macro preferred locations using spectral and force-directed methods, considering fixed components and ports.

\section{PRELIMINARIES}

\section{A. Problem Definition}

The input of macro placement is a netlist $N$ with hierarchy and array information derived from the RTL specification, and a fixed width and height. The outputs are the coordinates and orientation of each macro. The techniques presented in this work explicitly aim to obtain floorplans that show:

1) No overlaps between macro components.

2) Small wirelength, worst and total negative slack.

3) Macros being close to predefined preferred locations.

The first item guarantees that the layout can be used at late stages of the physical design flow, and is considered a priority in our approach. The second includes the primary metrics that define the global quality of the solution. The introduction of the dataflow affinity metric between blocks aims to capture connection bitwidth and register pipeline latency to reduce wirelenght and get good timing. Other metrics such as congestion are also reported in the experiments section but are not directly considered for optimization. The last item helps reduce variability, increase solution quality and provides additional control of the result of the algorithm to the physical engineers.

\section{B. Block Representation}

Each block represents the standard cells and macros under a hierarchy level, and has properties of both kinds of components. Given that our hierarchical top-down approach first decides block locations and later the position of the elements inside each block, cell area requirements and the possible slicing floorplans of the macros in a block are key to ensure that enough space is allocated in both dimensions when fixing the block area.

Formally, a block is characterized by a triplet $\left\langle\Gamma, a_{m}, a_{t}\right\rangle . \Gamma$ is a shape curve [26] that contains a set of pairs (width, height) representing the smallest bounding boxes that can hold slicing placements of the macros in the block (standard cells are ignored for $\Gamma$ ). $a_{m}$ (minimum area) is the sum of the area of all macros and standard cells under the hierarchy level. $a_{t}$ is the target area for the block, i.e., $a_{m}$ plus some extra area associated to the block (see Sect. IV-C).

Fig. 3 provides a visual reference of the block representation. Fig. 3a shows an abstraction of a block. The darker 


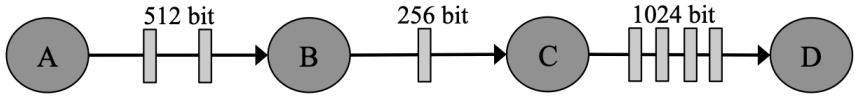

Fig. 4: Block connection graphs for a small system.

TABLE I: Data structures for different circuit abstractions.

\begin{tabular}{|ccll|}
\hline Graph & Size & Type of vertices & Description \\
\hline$H T$ & - & Hierarchical blocks. & $\begin{array}{l}\text { Circuit hierarchy } \\
\text { representation. }\end{array}$ \\
\hline$G_{n e t}$ & $\sim 10^{7}$ & $\begin{array}{l}\text { Macros, ports, sequential } \\
\text { and combinational cells. }\end{array}$ & $\begin{array}{l}\text { Bit-level complete } \\
\text { netlist connectivity. }\end{array}$ \\
\hline$G_{s e q}$ & $\sim 10^{5}$ & $\begin{array}{l}\text { Macros, multi-bit ports } \\
\text { and registers. }\end{array}$ & $\begin{array}{l}\text { Multi-bit sequential } \\
\text { connectivity. }\end{array}$ \\
\hline$G_{d f}$ & $\sim 10^{2}$ & Blocks and multi-bit ports. & $\begin{array}{l}\text { Dataflow affinity among } \\
\text { blocks and ports. }\end{array}$ \\
\hline
\end{tabular}

boxes represent macros. The blue rectangle $\left(a_{m}\right)$ represents the minimum area of the block, whereas the red rectangle $\left(a_{t}\right)$ represents the target area. Fig. $3 \mathrm{~b}$ shows its shape curve, defined by a set of Pareto points (in red). Given a rectangle for the block of dimensions $x_{r}$ and $y_{r}$, there is a possible placement of our macros in it if the point $\left(x_{r}, y_{r}\right)$ falls in the blue area. This area represents the space of rectangles that could hold a placement of the eight macros of the block.

\section{Dataflow Affinity}

For every pair of blocks $A$ and $B$, the dataflow affinity metric captures the "information closeness" between them. It is combined with physical distances to provide layout quality metrics. The dataflow affinity between two blocks varies with the number of bits and register pipeline stages between them, following the relation

$$
\text { Dataflow_Affinity }(A, B) \propto \frac{\text { Information_Flow }(A, B)}{\operatorname{Latency}(A, B)} .
$$

The motivation is that blocks with a high information flow have high dataflow affinity if their latency is small. Array width information at RTL is used to model information flow (bit count), and the number of sequential elements in paths between the blocks to model latency (depth of registers from one block to the other). An example is illustrated in Fig. 4 . showing a small system with 4 blocks and the connections between them. Some of these connections are critical because of the bitcount (D to C), and some because of the low latency (B to C). Blocks with high dataflow affinity need to be close in the layout.

\section{Circuit Abstractions}

Three graphs and a tree are used to model circuit connectivity (Table [ and Fig. 5). The netlist graph $G_{n e t}$ contains the lowest level of abstraction of the circuit (standard cell level). $G_{s e q}$ and $G_{d f}$ are subsequently derived from $G_{n e t}$ in order to model other views of the circuit considering multibit sequential elements (medium abstraction, Fig. 5b) and hierarchical block information (highest abstraction, Fig. 5c). The details of the obtention of $G_{s e q}$ and $G_{d f}$ from $G_{n e t}$ are presented in Sect. IV-D The hierarchical tree $H T$ (Fig. 5d,

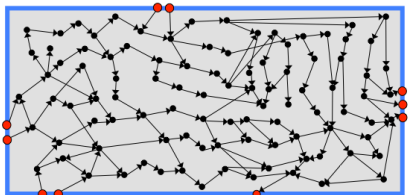

(a) Netlist graph $G_{n e t}$

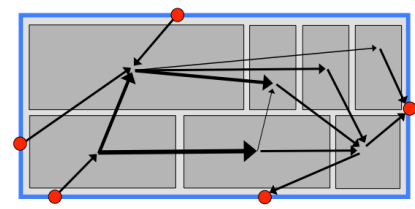

(c) Dataflow graph $G_{d f}$

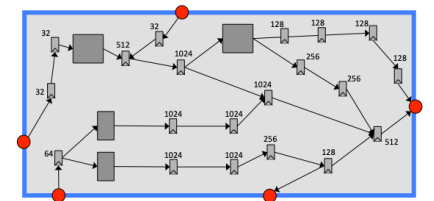

(b) Sequential graph $G_{s e q}$

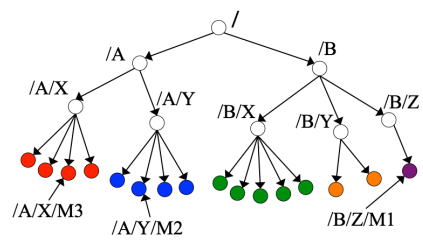

(d) Hierarchical Tree $H T$
Fig. 5: View of different circuit abstractions.

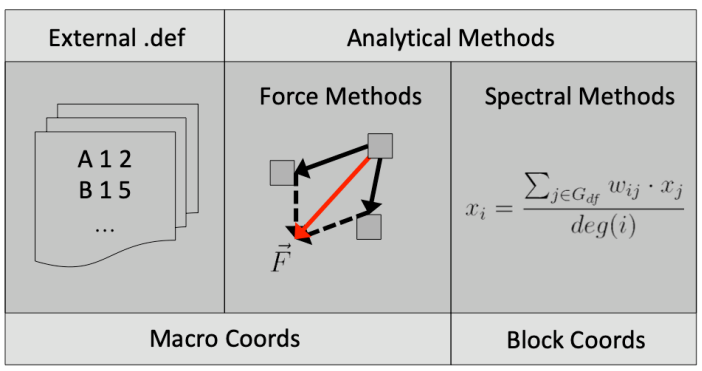

Fig. 6: Taxonomy of preferred location sources.

with some sample block and component names) is derived from the component names in the input hierarchical netlist $N$.

More formally, the graph $G_{n e t}=\left(V_{n e t}, E_{n e t}\right)$ represents the original netlist, with $V_{\text {net }}=M \cup P \cup F \cup C$, respectively being macros, ports, sequential cells (flops) and combinational cells.

The sequential graph $G_{s e q}=\left(V_{\text {seq }}, E_{\text {seq }}\right)$ is a directed graph with weighted nodes and edges. $V_{\text {seq }}$ represents macros, multibit registers and ports. Edges capture communication latency and array width between sequential components and ports.

The dataflow graph $G_{d f}=\left(V_{d f}, E_{d f}\right)$ is a directed graph with weighted nodes and edges that represents dataflow affinity, with $V_{d f}=B \cup P_{m b}$. Every node in $B$ represents a block and is formed by a subset of nodes in $V_{s e q}$. Nodes in $P_{m b}$ represent multi-bit ports. The edges hold information on the width and latency of the paths between the subsets of nodes in $V_{\text {seq }}$.

In $H T=\left(V_{h t}, E_{h t}\right)$, every node represents a level in the hierarchy, and edges represent subhierarchy relations. A hierarchy cut $H C$ with regard to a node $n \in V_{h t}$ is defined as a set of nodes in its subtree such that each path between $n$ and the leaves of its subtree crosses exactly one node in $H C$.

\section{E. Preferred Location Management}

HiDaP considers preferred macro locations by reducing the distances in the solution to these suggested locations. Fig. 6 shows the three proposed methods to provide preferred coordinates. They can be given at two levels: macro locations or block locations. The simulated annealing algorithm always works at a block level, but these blocks contain macros. If block coordinates are provided, they are directly used during the simulated annealing stage. If macro coordinates are provided, the center of gravity of the location of all macros inside the block is computed to find the preferred block location. 
The source of these coordinates can be threefold: and external def file, force-directed or spectral methods. The first two correspond to macro coordinates and propose a global suggestion for the entire macro placement problem, whereas the last provides block coordinates directly. The details of the automatic methods are given in Sect. VI Succinctly,

1) Spectral methods are executed at each multi-level instance of simulated annealing and find the coordinates for each block based on the dataflow graph $G_{d f}$ of the instance.

2) Force-directed methods rely on placing the sequential graph $G_{s e q}$ at the beginning of HiDaP execution, by obtaining an initial solution using spectral methods but refining it using force algorithms to reduce macro overlap.

\section{Algorithmic Overview}

HiDaP follows a multi-level flow by considering the hierarchy as a clustering of the design and using a decluster-andfloorplan scheme. Algorithm 1 provides a high-level view of the flow of the tool. The input is the hierarchical netlist $N$ and the dimensions of the circuit. The first step is the generation of the hierarchical tree from the netlist. shape_curve_generation constructs the set of shape curves $S_{\Gamma}$ which contains, for each $n_{h} \in V_{h t}$, a shape curve with the minimal sizes such that its macros can be placed inside under a slicing structure. Forcedirected methods are computed afterwards, once for the whole execution. The next steps are finding macro locations (recursive_block_floorplan) and orientations (memory_flipping), with a flipping post-process for wirelength reduction based on computing the dataflow affinity between macro corners.
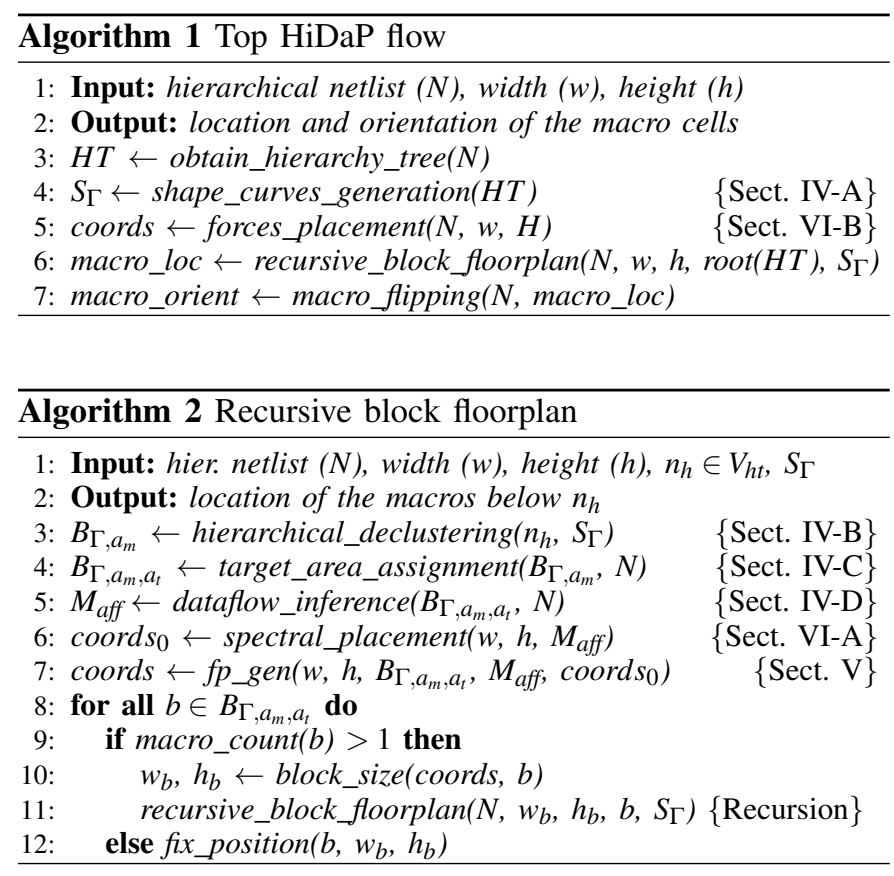

The recursive floorplanning function (Algorithm 2 ) receives, as inputs, the netlist $N$, a hierarchical node $n_{h} \in V_{h t}$, whose subtree must be placed in a given space $(w, h)$, and the shape curves for all hierarchical levels in $S_{\Gamma}$. The aim is to place the macros under $n_{h}$ considering dataflow between themselves and

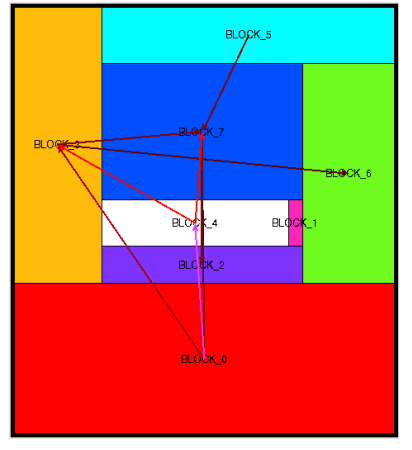

(a) Top hier. blocks layout

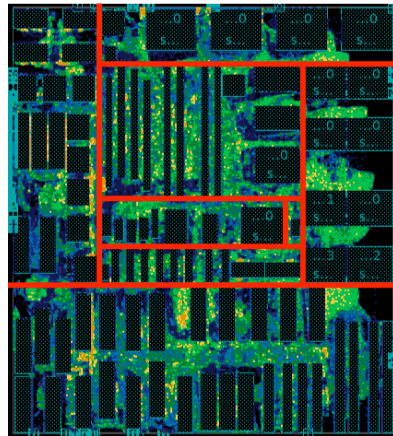

(b) Final macro placement
Fig. 7: Multi-level flow in HiDaP.

standard cell logic under $n_{h}$, but also (crucially) with macros outside $n_{h}$ and ports, to ensure a global view of the problem is kept at each floorplanning instance.

The first step is hierarchical_declustering, which identifies the blocks to be considered for floorplanning and partially characterizes them in the set $B_{\Gamma, a_{m}}$, with shape curve $\Gamma$ and the minimum area $a_{m}$ of each block. In target_area_assignment, the target area $a_{t}$ of the blocks is stored in $B_{\Gamma, a_{m}, a_{t}}$, a set containing the full characterization $\left\langle\Gamma, a_{m}, a_{t}\right\rangle$ of each block.

In dataflow_inference, the dataflow affinity between each pair of blocks is computed and stored in the affinity matrix $M_{a f f}$ and in spectral_placement, the preferred block location according to the available space, considering dataflow relations and allowing block overlap, is computed. During $f p \_g e n$ (floorplan generation), slicing structures are used to model the floorplan. Given preferred locations, from analytical methods or user suggested, and $M_{a f f}$, an optimization process (simulated annealing) is used to minimize three components: the product of the distance between blocks and their dataflow affinity, macro overlaps and distance to the preferred locations. After all block coordinates are fixed in coords, the process is recursively called for each block that has more than a single macro. Otherwise, its macro position is fixed in the corner of the available area that minimizes wirelength.

The example in Fig. 7 illustrates the multi-level nature of our approach. To the left (Fig. 7a, the layout of the top hierarchical blocks (obtained using Algorithm 2) is shown. Each colored box represents a hierarchical level (with macros and std. cells), and arrows between them indicate significant dataflow. After this layout if fixed, the hierarchical levels inside each one of the colored boxes are recursively placed, until the final macro placement is reached (Fig. 7b)

\section{Algorithmic Details}

\section{A. Shape Curves Generation}

Our hierarchical flow first decides the area allotted for a given hierarchical block, and then proceeds to find the location of its macros recursively. It is necessary to ensure at each hierarchical level that enough space is reserved to fit a slicing floorplan of all its macros, or overlaps would be introduced (more detail is presented in Sect.V). This initial step computes such feasible slicing floorplans for each hierarhy level and stores them in shape curves to be reused through the rest of the execution. 


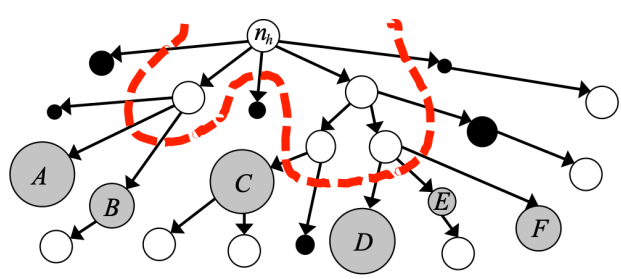

Fig. 8: Hierarchical declustering to find $H C_{B}$ and $H C_{G}$.

The input of this step is the hierarchical tree $H T$, and the output is a set of shapes curves $S_{\Gamma}$ where each $\Gamma$ is associated to a node in $V_{h t}$ describing possible sizes for the floorplan of the macros under its subtree. The subtree shape curves are calculated in a bottom-up fashion up to the root of the hierarchical tree. At the leaf nodes of $H T$, the associated $\Gamma$ contains the possible shapes of its macro. At each intermediate node of the hierarchy, it is not possible to compose the shapes of their children because the tree is not slicing. Since there is no general mode to compose the shape curves of a nonbinary tree, area-optimization floorplanning using simulated annealing generates a set of shape combinations with small area which are valid for the node.

\section{B. Hierarchical declustering}

The purpose of hierarchical declustering is to find the set of blocks to be considered during the floorplan of a given hierarchy level $n_{h}$, and to characterize their shape curve $\Gamma$ and minimum area $a_{m}$. The idea is represented in Fig. 8 . which shows the hierarchy tree under a node $n$. A possible hierarchical cut $H C$ with respect to that node (defined in Sec. [II-D, marked by a red line, generates block candidates for floorplanning. The nodes are divided in two sets, depending on the number of macros and area in their subtrees, and those with big area or with macros are selected (grey).

Formally, consider functions area(n) and macro_count( $n$ ), returning the sum of the area and macro count of the subtree rooted at $n \in V_{h t}$. Given a parameter defining a minimum area, the nodes in any hierarchy tree cut $H C$ can be divided in two sets, $H C_{B}$ and $H C_{G}$ :

$$
\begin{aligned}
& H C_{B}=\{n \in H C \mid \text { area }(n)>\text { min_area } \vee \text { macro_count }(n)>0\} \\
& H C_{G}=H C \backslash H C_{B} \quad \text { (small nodes with glue logic) }
\end{aligned}
$$

Nodes in $H C_{B}$ represent hierarchy levels with relatively big area or containing macros, whereas all others $\left(H C_{G}\right)$ are small nodes with glue logic. Each node in $H C_{B}$ is modeled as a block during layout generation. The nodes in $H C_{G}$ will be later absorbed by nodes in $H C_{B}$, as they represent small unstructured components.

The strategy used to find sets $H C_{B}$ and $H C_{G}$ for a given node $n$ is shown in Algorithm 3 . The parameter open_area is an area amount that controls how deep the tree will be explored, and min_area the minimum size for blocks to not be added to $H C_{G}$. Both open_area and min_area are a fraction of area $\left(n_{h}\right), 40 \%$ and $1 \%$ in the experiments.

\section{Target Area Assignment}

Since small and unstructured blocks in $H C_{G}$ are not directly considered for floorplanning, the goal of target area assignment
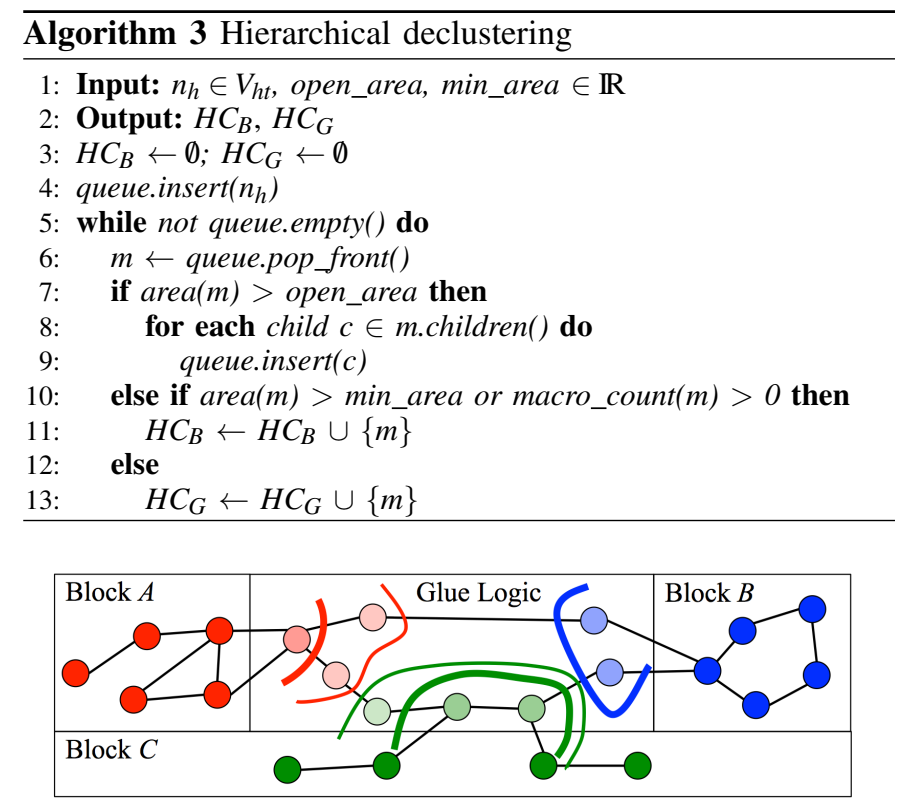

Fig. 9: Assigning $H C_{G}$ area to $H C_{B}$ blocks.

is to incorporate their area to the target area $a_{t}$ of blocks in $H C_{B}$. In $G_{n e t}$, a multi-source breadth-first search [29] finds the shortest paths from all nodes in $H C_{B}$ to any cell in $H C_{G}$. Fig. 9 shows three blocks in $H C_{B}$ with fully painted nodes, connected by glue logic components (elements in $H C_{G}$ blocks) which are incorporated to their closest blocks as they are reached during the search. After this process, the sum of the area of $H C_{B}$ blocks represents the whole area of the floorplanning instance and the triplet $\left\langle\Gamma, a_{m}, a_{t}\right\rangle$ for each block is characterized.

\section{Dataflow Inference}

Dataflow inference generates an affinity matrix $M_{\text {aff }}$ estimating the affinity between each pair of blocks, ports and other macros in the circuit. $M_{\text {aff }}$ is derived from the adjacency matrix of the dataflow graph $G_{d f}$, which is obtained from $G_{\text {seq }}$ and $G_{n e t}$. First $G_{n e t}$ is transformed into $G_{s e q}$ in the following steps:

1) $C$ nodes representing combinational cells are removed by connecting their predecessors to their successors

2) Nodes in $P$ and $F$ are clustered using component names to find array structures (name $[n]$, name_n).

3) Edges between sequential components are inferred by analyzing their transitive fanin/fanout in $G_{n e t}$ and discovering their paths.

4) To reduce graph size but keep relatively big components, elements with fewer bits than a threshold are discarded.

The next step is constructing the dataflow graph $G_{d f}$ from $G_{s e q}$. Each node in $V_{d f}$ represents a hierarchical block, which is associated to a set of nodes from $V_{\text {seq }}$. Connectivity information of edge $e_{i, j}$ in $E_{d f}$ takes the form of a histogram, where bins represent latency and their height represents number of bits. A breadth-first search at $G_{s e q}$ starts simultaneously from all components of block $i$ traversing only outgoing edges through glue logic. When a component of block $j$ is reached, the bitwidth of its predecessor in the path is added to the bin corresponding to the number of flops stages. 
In order to condense the dataflow information of the histogram between two blocks, the weight of their edge is computed using $\operatorname{score}(h, k)$ for an integer $k$ according to the following formula, where $i$ indexes bins in the histogram:

$$
\sum_{i} \# b i t s_{i} / \text { flop_stages }{ }_{i}^{k}
$$

The formula is based in the relation presented in Sec. III-C Parameter $k$ controls the exponential decay impact of latency.

\section{LAYOUT GENERATION}

The goal of layout generation is, given the block characterizations and their dataflow affinity relation (as computed in Sect. IV-B to Sect. IV-D], to a block layout with reduced wirelength, timing, overlaps the distance between macros and their preferred locations.

The layout is represented as a slicing tree with a node per block. This structure allows to work with shape curve compositions and naturally use a top-down algorithm for layout generation. The solution space is explored using simulated annealing. The structure is implemented using a reverse polish expression which is perturbed with equal probability with one of three operations: operand swap, operator inversion or operand-operator swap (similar to [33]).

\section{A. Objective function}

As exposed in Sect. II-A our method proposes to find layouts that minimize several metrics at the same time: wirelength and timing, distances to proposed locations and overlaps. The proposed cost function to minimize is:

$$
\mu_{1} \times \text { DATAFLOW }+\mu_{2} \times \text { DISTANCE }+\mu_{3} \times O V E R L A P
$$

The parameters $\mu_{1}, \mu_{2}, \mu_{3}$ adjust the weights of the components and are automatically updated during the simulated annealing execution as explained in Section V-B DATAFLOW represents, for each pair of blocks $b_{i}, b_{j}$, the sum of the products of their dataflow affinity and distance,

$$
\sum_{b_{i}, b_{j}} \text { dataflow_affinity }\left(b_{i}, b_{j}\right) \times \operatorname{distance}\left(b_{i}, b_{j}\right) .
$$

Its minimization ensures that blocks that have large array connections and relatively small latency are close in the layout, aiming at obtaining good properties in terms of wirelength and timing. The position of ports and macros outside the subtree are considered a fixed point during the computation.

The second term DISTANCE represents the displacement between the block in the layout and the preferred location of its components. In the case of blocks for which more than one macro location is proposed, the center of gravity is computed and used to compute the distance with the current block location.

Finally, the OVERLAP term represents the sum of overlapping areas in the layout multiplied by a constant that increases with the severity of each illegality. This mechanism is used to ensure macro overlaps penalize more than standard cell area overlaps.

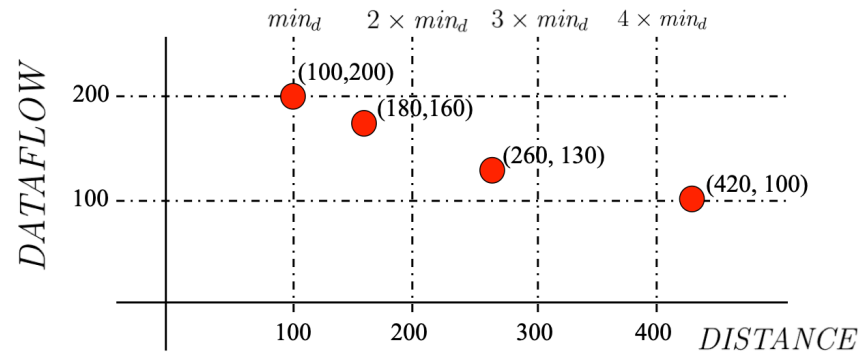

Fig. 10: Example of a Pareto-optimal set of solutions.

\section{B. Adaptive Parameters}

The adaptive parameters in the objective function $\left(\mu_{1}, \mu_{2}, \mu_{3}\right)$ automatically adjust the relative weight of the components during the exploration. This allows the algorithm to adapt to all possible designs and sub-problems: if the weights were fixed, they would need to be hand-tuned for each new instance according to the relative amounts of each component in the function.

In order to control the multi-objective optimization, the user does not directly indicate $\mu_{1}, \mu_{2}, \mu_{3}$, but the desired relative weights for each one of the components of the formula: $\alpha_{1}, \alpha_{2}, \alpha_{3}$ (with $\alpha_{i} \in[0,1], \alpha_{1}+\alpha_{2}+\alpha_{3}=1$ ). At each temperature change in the simulated annealing, the values of $\mu_{1}, \mu_{2}, \mu_{3}$ are updated to ensure their components have the chosen relative weight following the formula

$$
\mu_{i}=\left(\alpha_{i} \times \text { mean_total_cost }\right) / m e a n \_c o m p o n e n t \_c o s t .
$$

The mean costs are calculated over all explored solutions since the last temperature change. The variation of each $\mu_{i}$ is limited at each iteration, to ensure the multipliers do not change sharply and trap the layout in an early local minimum.

\section{Keeping the best solution}

Our approach includes a mechanism to keep the best solution visited during the search. The best solution is always the most legal, and a trade-off is used to balance the final solution in terms of DATAFLOW and DISTANCE. To do so, the most legal solutions are stored as a set of Pareto points in two dimensions, DATAFLOW and DISTANCE of the solution. The points are only stored if their OVERLAP value is equal to the lowest found until the point, ensuring the best solution is always the most legal. At the end of the search, given the lowest found DISTANCE score min_d, the solution with a DISTANCE equal or lesser to $\gamma \times \min \_d$ with the lowest DATAFLOW is considered the best solution $(\gamma \geq 1$ is a userdefined parameter).

The mechanism is illustrated in Fig. 10. It depicts a set of candidate layouts where the $x, y$ axis represent DISTANCE and DATAFLOW, and each point is one of the most legal Paretooptimal solution at the end of a simulated annealing search. If the user-configurable parameter $\gamma$ is 1 , the best solution is the one where DISTANCE $=100$, DATAFLOW $=200$. But if more flexibility is allowed in DISTANCE minimization $(\gamma=2)$, a slightly better solution in terms of DATAFLOW can be found $(180,160)$. Finally, if a much greater $\gamma$ is allowed, the solution that best minimizes DATAFLOW is $(420,100)$. 


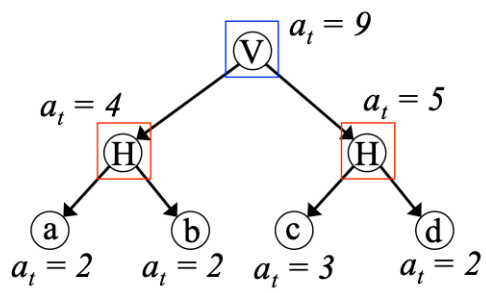

(a) Slicing Tree representation.

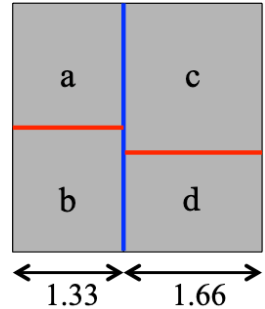

(b) Resuting layout.
Fig. 11: Recursive layout generation.

\section{Layout Representation and Search}

This section describes the algorithm to decide the coordinates and sizes of each block from a given slicing tree. There is a well-known method to obtain a layout from a slicing tree which consists of composing, at each node, the shape curves of its two children according to its operator. It has been widely studied in the context of non-fixed outline floorplans (where area minimization is one of the objectives for the formulation). For fixed-outline floorplanning, the approach presents two limitations:

- If the sum of the whole floorplan is divided among all placeable components, it is hard to find a valid floorplan that fits exactly in the allotted area. Extra legalization steps are necessary to redistribute whitespace.

- Module shapes must be discretized beforehand. Blocks with standard cells must choose from a set of aspect ratios: too few result in bad solution space exploration, too many increase computation time greatly.

To overcome the first, our approach considers the allowed floorplan area as a budget: the layout always takes exactly the area it has been assigned. To overcome the second, and in combination with this notion, a top-down approach is proposed to determine the coordinates of each block. Using this method, it is important to check that macros under each hierarchy block fit using a slicing structure in its assigned area by using the shape curves computed in Sect IV-A

The following example illustrates the algorithm in the case of standard cell blocks only. Let $\Gamma^{n}, a_{m}^{n}$ and $a_{t}^{n}$, for a given slicing tree node $n$, characterize its subtree as a block. They are computed at the beginning of the layout generation from the blocks at the leaves by composing shape curves and adding areas up to the root, similarly to the well-known "slicing tree to layout" algorithm. The top-down process begins at the root, where the entire area is available. At each node, the available area is partitioned vertically or horizontally (depending on the node operator), proportionally to the target area $a_{t}$ sum of its subtrees. The process continues until the leaves are reached, assigning a rectangle to each block.

Fig. 11 shows a slicing tree where each leaf has target area $a_{t}$, and its layout considering a budget of $3 \times 3$ area units. Beginning from the leaves, the $a_{t}$ 's of all nodes are computed up to the root. The first partition, the vertical blue line from the $V$ cut in the root, divides the horizontal budget among its children, with $a_{t} 4$ and 5 . The process continues recursively with the $H$ cuts until the leaves are reached and the rectangular layout for each block has been decided.
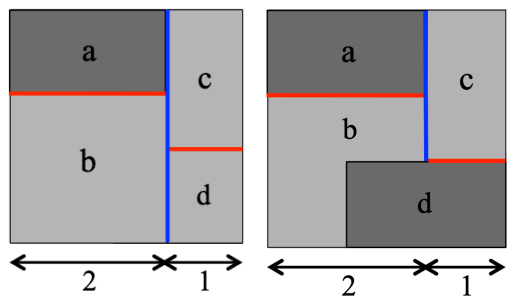

(a) Macro in $a$. (b) Macros in $a, d$.

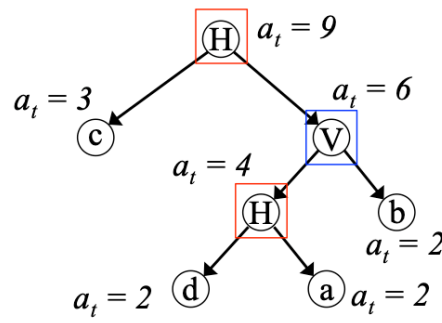

(d) Slicing Tree for (c).

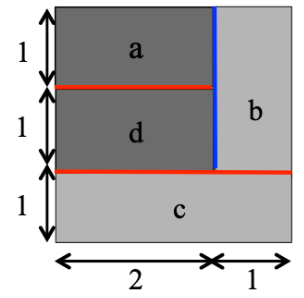

(c) Legal layout.
Fig. 12: Handling of macros in blocks.

\section{E. Slicing Tree to Layout: Detailed View}

The presented technique guarantees that the $a_{t}$ demands for every block are met. Still, the layouts of some blocks may be illegal when they include macros. It would be the case of node $a$ if it would include a macro with $w=2$ and $h=1$, since such macro would not fit in the allotted space in the example. The situation is shown in Fig. 12a It presents the same case shown in Fig. 11, but now the $\Gamma$ of block $a$ indicates it contains a macro with size $2 \times 1$. Given the minimum width is 2 for the block, the constraint is temporarily solved by pushing the cut from 1.33 to 2 (according to the $a_{t}$ of the subtrees), at the cost of stealing area of blocks $c$ and $d$ and slightly increasing the $O V E R L A P$ factor of the cost function. If additionally block $d$ contains a macro of the same size, the sum of both macro widths in this cut is 4 and both macro sizing requirements can not be satisfied at the same time. In this case, the cut is done according to one of them, and the OVERLAP increases severely. A possible solution without OVERLAP penalization is shown in Fig. 12c deriving from the alternative slicing tree shown in Fig. $12 \mathrm{~d}$

The minimum width of the macros when doing a $V$ cut in a hierarchical block is computed by examining their shape curve. Given that the maximum $h$ for the macros is known, the tool finds the minimum $w$ such that there exists a point $(x, y)$ in the shape curve with $x \leq w$ and $y \leq h$, guaranteeing all macros for the block to fit in said space. Consider the example $\Gamma$ shown in Fig. 12e This block has two possible macro shapes, $(2.5,5)$ and $(5.5,5)$, but given for example a fixed height at 2 , the minimum width for the macros must be 5.5 .

\section{Vi. Generation of Preferred Macro Locations}

The generation of preferred macro locations can be also automated. Our proposal is to use analytical methods, in particular spectral and force-directed methods, to compute these locations. Spectral methods can be used to determine the location of each block in a particular simulated annealing 
instance. However, when applying spectral methods at the beginning of the HiDaP execution to the sequential graph to obtain a global view of the macro preferred locations, the components are not spread enough. Force-directed methods are applied to obtain a better macro distribution.

\section{A. Spectral Method for the Dataflow Graph}

The input to the spectral placement is the rectangle layout and the dataflow graph $G_{d f}$. The output is a location for each block inside the rectangle. The coordinates of ports and macros are also provided to consider their location, possibly outside the placement region. The implemented algorithm is a modified version of the spectral graph drawing method proposed by Koren [20], which is computationally fast and can be extended to handle fixed ports.

Using degree-normalized eigenvectors avoids the situation in which dense clusters are drawn very densely and the rest of the area is not well used, which happens when minimizing wirelength without considering whitespace distribution. The key observation in [20] is that the optimal position for node $i$ to minimize the sum of squared wirelengths is, with $w_{i j}$ being the edge weight between nodes $i$ and $j$, the weighted centroid of $i$ 's neighbors,

$$
x(i)=\frac{\sum_{\langle i, j\rangle \in E} w_{i j} x(j)}{\operatorname{deg}(i)}
$$

The algorithm to compute degree-normalized eigenvectors relies on this fact to iteratively perform this product with other small adjustments until convergence is reached. Our extension of the original algorithm consists in the incorporation of fixed nodes (ports and macros outside our subproblem) to the formulation. After each iteration, the location of the fixed nodes is updated with the known positions. All nodes which are not fixed begin at a random position and converge to their ideal position. In our use case, the number of nodes is small, usually less than 30 .

\section{B. Force-Directed Method for the Sequential Graph}

The force-directed placement is applied to the sequential graph $G_{s e q}$ instead of the dataflow graph $G_{d f}$. As $G_{s e q}$ has thousands of nodes, the spectral method neither produces enough spreading, nor provides good mechanisms to avoid macro overlap. The force-directed method is applied once at the beginning of the flow and macro positions are later used in the corresponding block floorplanning problems during the whole execution of HiDaP.

The spectral method positions of $G_{s e q}$ are refined by graphdrawing algorithms based on well-known spring methods [15], [32]. In these methods the edges represent springs, the nodes are placed in a two-dimensional layout and the system is released. The springs move the nodes to find the minimal energy state where they are in equilibrium. The corresponding spring force depends on the edge weight, thus having pairs of nodes with higher connectivity closer in the layout. To suit our particular placement needs, a macro overlap removal force has been added to the approach.
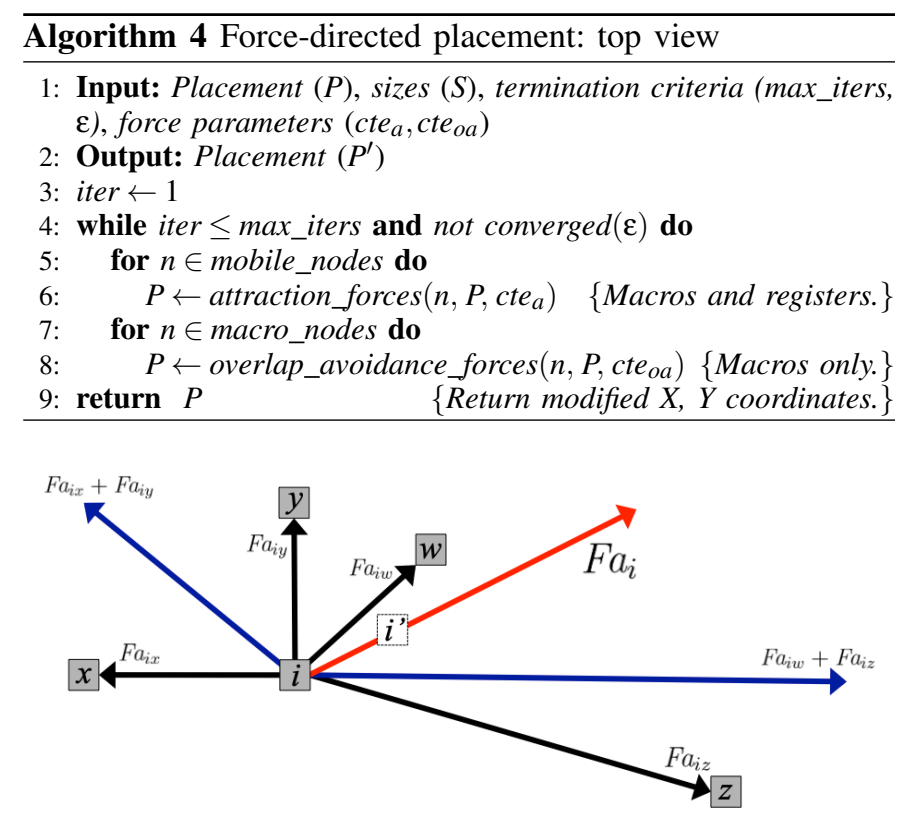

Fig. 13: Example of attraction forces.

The top view of our force-directed sequential placement algorithm is shown in Algorithm 4 The inputs are the placement $P$ with the $X, Y$ coordinates from spectral placement and the vector $S$ with component sizes, termination criteria parameters max_iters, $\varepsilon$ and user-defined force parameters cte $_{a}$, cte $_{o a}$ to control the impact of the attraction and overlap-avoidance forces. From the positions in the initial placement $P$, while the number of iterations has not exceeded max_iters and the system is not stable, all mobile nodes (registers and macros) are moved sequentially according to their attraction forces (i.e., edges to other components), and all macros are moved to try to reduce overlap according to the overlap-avoidance forces. At the end of the process, the modified placement coordinates for all components are returned.

\section{Computation of attraction forces.}

The basic idea for the action of attractive forces is shown in Fig. 13. Each node $i$ (gray boxes) is attracted to all its neighbors $j$ with a force $F a_{i j}$ depending on their distance and edge weight $w_{i j}$ (black arrows). All of these attractive forces are added (blue arrows) and the resultant force $F a_{i}$ (red arrow) is computed. The node $i$ moves a certain distance in this direction, proportionally to cte $_{a}$ (new position is marked by the white box). The movement in any direction is halted if the node is to leave the placement area.

\section{Computation of forces to avoid overlap.}

The motivation of overlap-avoidance forces is to push macros to positions where they reduce the overlap with other macros. For each overlapping macro, neighboring locations are scanned and its overlap with other macros is computed. The macro moves in the direction of the position with least overlap and closest to its own location, by an amount proportional to the parameter cte $_{\text {oa }}$. If the macro has no overlaps, it has no motivation to move. The algorithm is essentially quadratic, in worst case it compares overlap for each pair of macros. It is feasible in reasonable time given that it is only applied 


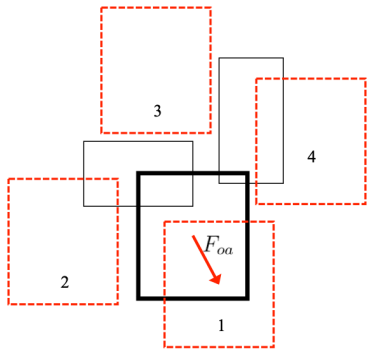

(a) Move towards less overlap.

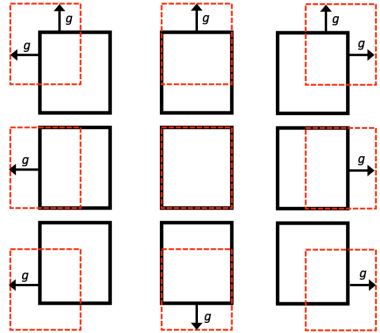

(b) Scan in grid around macro.
Fig. 14: Overlap-avoidance force

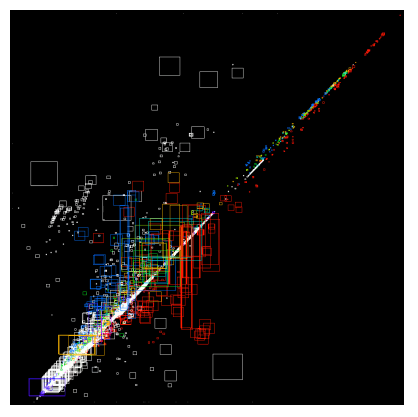

(a)

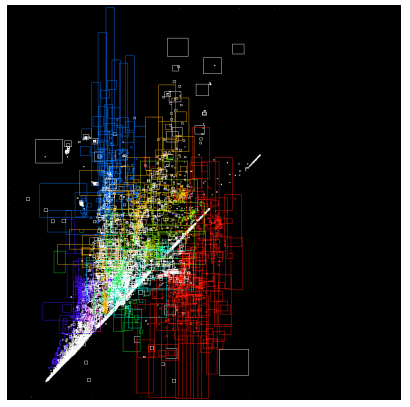

(b)
Fig. 15: Evolution of the force-directed algorithm.

to macro objects and only considers a restricted local area. The idea is illustrated in Fig. 14a. The black boxes represent macros (with some overlaps), and the process focuses on the one with wider border. Several candidate locations are considered ( 1 to 4 , in red), and the amount of overlap against other macros is computed. Finally the $F_{o a}$ force goes towards 1, which has no overlap with other macros and is closer than 3 .

In order to find candidate positions to reduce overlap, a grid scan is executed centered at the target macro location. Given the window_size and grid_unit parameters, the considered positions are a quadratic search around the macro, with $x$ position from $x$-window_size to $x+$ window_size, step grid_unit, and simultaneously $y$-window_size to $y+$ window_size, step grid_unit. An example is shown in Fig. 14b with some grid_unit $=g$ and window_size $=1$. In practice, grid_unit is a fraction (tuned by user parameter grid_size_factor) of the area of the smallest macro, ignoring aspect ratios,

$$
\text { grid_unit }=\min _{i \in \text { macros }} \sqrt{\frac{w_{i} \times h_{i}}{\text { grid_size_factor }}}
$$

Additionally, to make the process happen smoothly, macro size scales as iteration count advances, from points at iter $=1$ to their full size at iter = max_iters, and overlapping with canvas borders or outside implies infinite overlap.

An example of the evolution of the execution of the forcedirected algorithm is shown in Fig. 15. Rectangles represent macros and registers, and their colors depends on their top hierarchical block. At the beginning (Fig. 15a) the positions come from spectral placement, which had certain spreading properties. By the end of the iteration count (Fig. 15b), the design is much more spread. Notice the final goal of our forcedirected placement is to obtain preferred macro locations for
TABLE II: Benchmark cell and macro count.

\begin{tabular}{|c|cc|c|cc|}
\hline Bench. & Cells & Macros & Bench. & Cells & Macros \\
\hline$c 1$ & $520 \times 10^{3}$ & 32 & $c 5$ & $1.39 \times 10^{6}$ & 133 \\
\hline$c 2$ & $3.95 \times 10^{6}$ & 100 & $c 6$ & $2.87 \times 10^{6}$ & 90 \\
\hline$c 3$ & $3.78 \times 10^{6}$ & 94 & $c 7$ & $1.67 \times 10^{6}$ & 108 \\
\hline$c 4$ & $4.81 \times 10^{6}$ & 122 & $c 8$ & $2.20 \times 10^{6}$ & 37 \\
\hline
\end{tabular}

the floorplanning algorithm of HiDaP: it is not to distribute the objects perfectly evenly and with zero overlap. The runtime for the biggest circuits is about 5 minutes.

\section{EXPERIMENTAL EVALUATION}

Array information for dataflow analysis is essential for the quality of results in our method. Unfortunately, this information is not present in open benchmarks available to academia such as the ICCAD'12 benchmarks [1]. For this reason, a set of 8 real industrial examples of challenging circuits on FinFET nodes (12 metal layers) were used to validate our approach, containing millions of gates and up to 133 macros (as shown in Table III). The final handcrafted layouts obtained by expert back-end engineers were available for comparison. The following five scenarios are evaluated and compared:

Industrial EDA (IndEDA) Floorplans obtained with a stateof-the-art industrial tool using high effort settings.

Handcrafted floorplan (handFP) Floorplans manually obtained by expert back-end engineers at the company.

RePlAce (RePlAce) Floorplans generated by RePlAce [10] (global placer in OpenROAD [4]). As they are not legalized, macro overlap removal is performed by IndEDA.

DATE results (DATE) Preliminary results reported in [30]. Best of three generated floorplans.

Our results (HiDaP) Results obtained using the approach presented in this paper.

Unless otherwise noted, metrics are measured after performing placement of standard cells with IndEDA. Wirelength and total negative slack averages are reported using the geometric mean to reduce sensitivity to extreme values. Wirelength is shown in meters, the worst negative slack is reported as a percentage of the clock period, and total negative slack is shown in ns (less is better). Given the stochastic nature of our algorithm, the HiDaP flow is run 5 times; the presented results are their average and minimum. The runtime of HiDaP ranges from 10 to 90 minutes depending on the macro count and hierarchy of each particular circuit.

\section{A. Results After Placement}

The plots in Fig. 16 compare the placement results for our benchmark suite. The reference values include handFP, IndEDA, RePIAce (one run each) and results from DATE [30] (left columns). Compared against them to the right are 2 versions of our tool. The blue columns represent using spectral methods on $G_{d f}$ (corresponding to Sect. VI-A) and keeping the average of 5 runs (HiDaP-SA) or minimum of 5 runs (HiDaP$\mathrm{SM})$. Yellow and orange columns represent using spectral and force-directed methods on $G_{s e q}$ (corresponding to Sect. VI-B) and keeping the average of 5 runs (HiDaP-FA) or minimum of 5 runs (HiDaP-FM). 

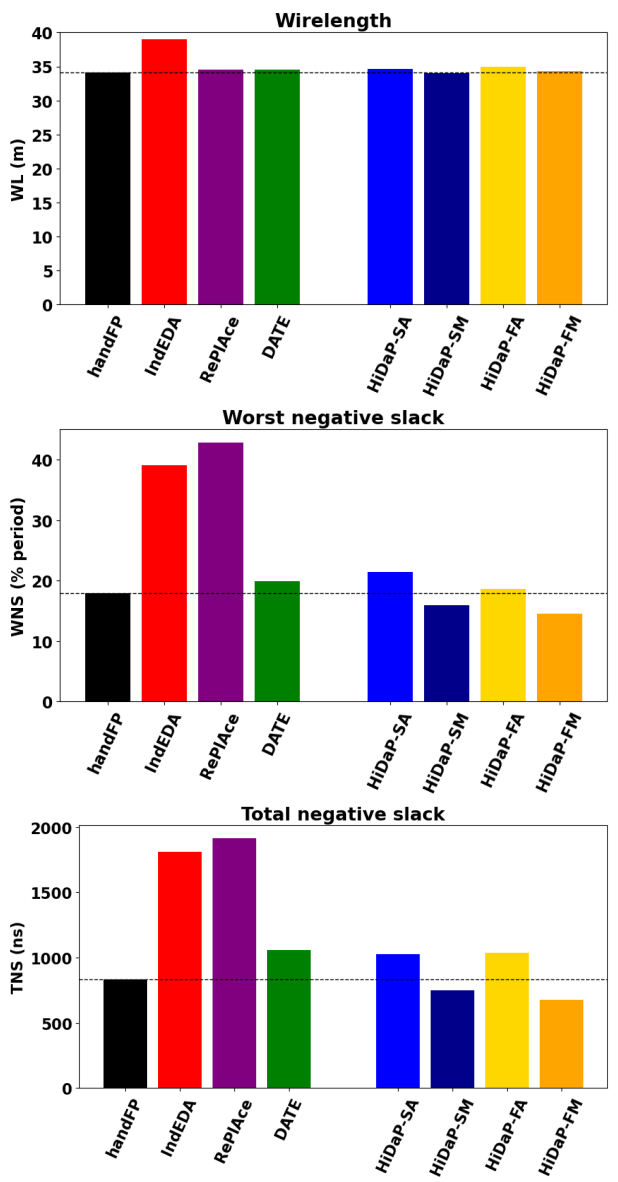

Fig. 16: Comparison of HiDaP to other approaches.

When comparing the automated flows against the manual handFP flow, IndEDA shows the least promising results, with worse numbers in all aspects (up to $14 \% \mathrm{WL}$ increase, and near double WNS and TNS). RePIAce offers much better wirelength on par with manual designs, but the timing results are slightly worse than IndEDA. The results reported using spectral and forces are very close to the ones from the handcrafted floorplans in all metrics. In particular, the wirelength when using spectral and forces methods remains very similar to the manual results and obtained with DATE. However from the timing perspective, using spectral and forces gives better results. In particular the results improve on the ones reported in [30], as taking the average of 5 (HiDaP-SA, HiDaP-FA) they are similar to DATE (which were a best of 3 ), and the current approach reports significantly lower TNS and WNS when taking the min of 5 runs per circuit (HiDaPSM, HiDaP-FM).

\section{B. Best Layouts After Placement}

The charts in Fig. 17 compare the average metrics (after placement) of the reference results against picking the best layout per circuit (the ones with best WL and TNS respectively) from a set of 40 runs per layout obtained by HiDaP, created using 8 parameter recipes and 5 seeds for each recipe. The average congestion of RePIAce nearly doubles that of manual designs, hinting why its wirelength results do no translate into timing gains. When focusing on the HiDaP layouts with best
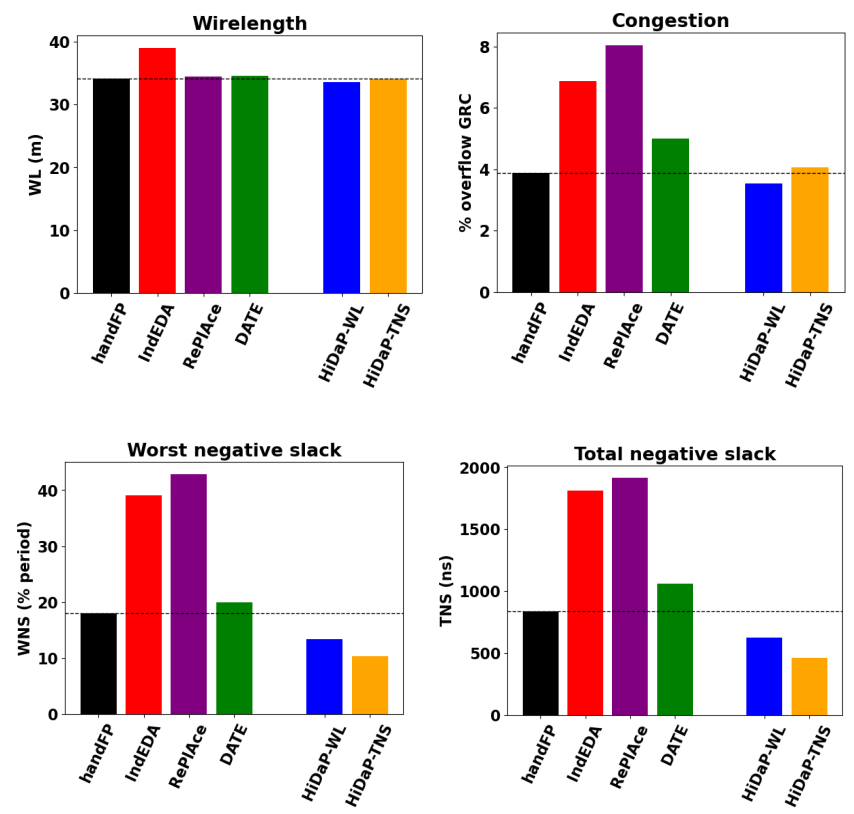

Fig. 17: Placement results, best layouts.

TABLE III: Effect of latency decay factor.

\begin{tabular}{|c|c|cc|rr|rr|}
\cline { 3 - 8 } \multicolumn{2}{c|}{} & \multicolumn{2}{c|}{ Wirelength } & \multicolumn{2}{c|}{ WNS } & \multicolumn{2}{c|}{ TNS } \\
\hline Flow & $k$ & \multicolumn{1}{c|}{$\mathrm{m}$} & Norm. & \multicolumn{1}{c|}{ \%clk } & \multicolumn{1}{c|}{ Incr. } & \multicolumn{1}{c|}{ ns } & Norm. \\
\hline handFP & - & 34.13 & 1.000 & -17.96 & $0 \%$ & -835 & 1.00 \\
\hline & 0 & 35.04 & 1.026 & -28.22 & $10.26 \%$ & -1393 & 1.67 \\
HiDaP & 1 & 34.57 & 1.013 & -23.38 & $5.42 \%$ & -1254 & 1.50 \\
& 2 & 34.86 & 1.021 & -21.95 & $3.99 \%$ & -1266 & 1.52 \\
\hline
\end{tabular}

TABLE IV: DATAFLOW and DISTANCE optimization tradeoff

\begin{tabular}{|c|cccc|}
\cline { 2 - 5 } \multicolumn{1}{c|}{} & \multicolumn{2}{c|}{$\gamma=\infty$} & \multicolumn{2}{c|}{$\gamma=1$} \\
\hline$\alpha_{1}, \alpha_{2}, \alpha_{3}$ & $(1)$ & $(2)$ & $(2)$ & $(3)$ \\
\hline DATAFLOW & 1.00 & 1.03 & 1.30 & 1.38 \\
\hline DISTANCE & 4.73 & 3.64 & 1.13 & 1.00 \\
\hline
\end{tabular}

WL reduction, WL drops by a $2 \%$ while WNS is reduced by close to a 5\% of cycle time and TNS is reduced by a $25 \%$ compared to handFP. When focusing on timing, WNS is reduced by close to a $8 \%$ of cycle time when compared to the handFP results, and the TNS is reduced by up to a $44 \%$, showing our tool can find very good layouts at the price of parallelizable CPU time.

\section{Effect of Latency Awareness}

The effect of the variation of the latency decay parameter $k$ (introduced in Sect. IV-D), affecting the computation of the edges weights in the dataflow graph, is shown in Table III A value of $k=0$ means considering all latencies to be 1 , whereas $k=2$ means considering them squared. All results are worse when ignoring flop pipeline stages, specially WNS, which is a $10 \%$ worse than handFP in percentage of cycle time. When increasing $k$, the best point for WL and TNS seems to be at $k=1$, although additional reduction in WNS can be achieved with $k=2$. This reinforces the idea that considering flop latencies between components is a key element to achieve floorplans close to timing sign-off.

\section{DATAFLOW-DISTANCE tradeoff}

When the engineer suggests locations to the tool, it is often the case that dataflow metric is negatively impacted in order 


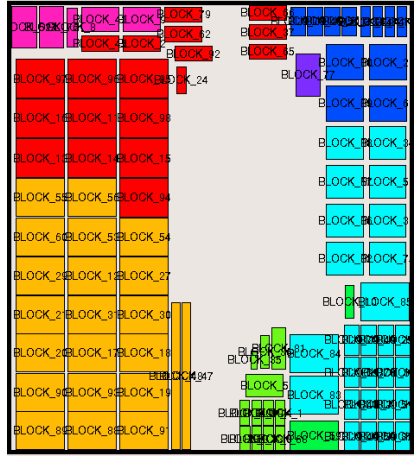

(a) Suggested macro locations

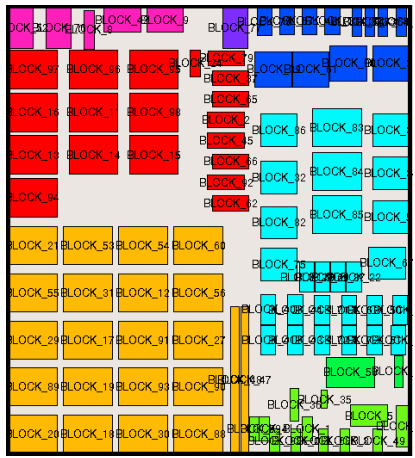

(b) Constrained floorplan
Fig. 18: Guiding HiDaP using known macro locations.

TABLE V: Geo. mean over the best layouts of each circuit after routing. The first two rows average over the eight circuits, the next three only over the six that IndEDA managed to route.

\begin{tabular}{|l|rr|r|rr|rr|}
\cline { 2 - 9 } \multicolumn{1}{c|}{} & \multicolumn{2}{c|}{ Wirelength } & \multicolumn{1}{c|}{ Legal } & \multicolumn{2}{c|}{ WNS } & \multicolumn{2}{c|}{ TNS } \\
\hline \multicolumn{1}{c|}{ Flow } & $\mathrm{m}$ & Norm. & \multicolumn{1}{c|}{ DRC } & \%clk & \multicolumn{1}{c|}{ Incr. } & \multicolumn{1}{c|}{ ns } & Norm. \\
\hline handFP & 34.27 & 1.000 & 1447 & -23.0 & $0 \%$ & -298 & 1.00 \\
HiDaP-M & 34.71 & 1.013 & 1561 & -16.5 & $-6.5 \%$ & -92 & 0.31 \\
\hline handFP & 33.42 & 1.000 & 1546 & -20.0 & $0 \%$ & -73 & 1.00 \\
IndEDA & 38.16 & 1.142 & 55288 & -19.5 & $-0.5 \%$ & -28 & 0.38 \\
HiDaP-M & 34.05 & 1.019 & 1720 & -15.7 & $-4.3 \%$ & -21 & 0.30 \\
\hline
\end{tabular}

to reduce distances to such locations. Table IV shows the impact on both components of the cost function in average across all circuits, 5 runs for each, when using the handFP solution to guide the search of the HiDaP flow. Columns represent 4 parameter recipes. Recall when $\gamma=\infty$, the result with lowest DATAFLOW is kept, and when $\gamma=1$, the result with lowest DISTANCE is kept. The values for $\alpha_{1}, \alpha_{2}, \alpha_{3}$, are $(1)=0.8,0.0,0.2$ (optimize only dataflow and overlap), $(2)=0.4,0.4,0.2$ (optimize all) and $(3)=0.0,0.8,0.2$ (optimize only distances and overlap).

When the optimization effort and best solution kept minimize dataflow $(\gamma=\infty, 1)$, the distance to desired positions is 4.73 times the minimum possible distance. On the other hand when minimizing distances to positions is prioritized, the DATAFLOW component is $38 \%$ higher. This tradeoff is not always existing: in $c 5$, both components can be simultaneously minimized as the handmade placement also minimized dataflow affinity. Fig. 18 shows the effect of preferred macro locations visually: the layout to the left is the handFP layout for $c 2$, whereas the layout to the right is the one obtained by HiDaP when given the handFP layout as a suggestion and enforcing distance minimization.

\section{E. Results After Routing}

Previous experiments were evaluated after placement, where an initial assessment of whether or not a macro placement is good enough can be made. The 5 layouts from Sect. VII-A that were obtained using spectral methods on $G_{d f}$ were also brought to routing in order to check the effects of congestion and ensure that quality was preserved. Table V shows a summary of the results, considering our best routed layout of the 5 under the name HiDaP-M.

Each line in Table $\mathrm{V}$ represents the average of the best layouts for each circuit. The first two lines average over
TABLE VI: Metrics after routing of layouts in Sec. VII-A

\begin{tabular}{|c|c|c|c|c|c|c|c|}
\hline Case & \multicolumn{2}{|c|}{ Wirelength } & Legal & \multicolumn{2}{|c|}{ WNS } & \multicolumn{2}{|c|}{ TNS } \\
\hline $\mathrm{c} 1$ & $\mathrm{~m}$ & Norm. & DRC & $\% \mathrm{clk}$ & Incr. & ns & Norm. \\
\hline handFP & 12.97 & 1.000 & 4 & 0 & $0 \%$ & -0.1 & 1.00 \\
\hline IndEDA & 14.98 & 1.155 & 1 & -1.4 & $1.4 \%$ & -0.2 & 2.00 \\
\hline HiDaP-M & 14.29 & 1.102 & 2 & -0.8 & $0.8 \%$ & -0.1 & 1.00 \\
\hline $\mathrm{c} 2$ & WL & Norm. & DRC & WNS & Incr. & TNS & Norm. \\
\hline handFP & 39.40 & 1.000 & 58 & -51.7 & $0 \%$ & -1760 & 1.00 \\
\hline IndEDA & 45.12 & 1.145 & $>10^{5}$ & -7.8 & $-43.9 \%$ & -4 & 0.00 \\
\hline HiDaP-M & 41.14 & 1.044 & 14 & -4.9 & $-46.8 \%$ & -3 & 0.00 \\
\hline $\mathrm{c} 3$ & WL & Norm. & DRC & WNS & Incr. & TNS & Norm. \\
\hline handFP & 38.27 & 1.000 & 30 & -5.8 & $0 \%$ & -8 & 1.00 \\
\hline IndEDA & 42.02 & 1.098 & $>10^{4}$ & -12.7 & $6.82 \%$ & -7 & 0.94 \\
\hline HiDaP-M & 36.34 & 0.950 & 37 & -15.0 & $9.2 \%$ & -13 & 1.66 \\
\hline $\mathrm{c} 4$ & WL & Norm. & DRC & WNS & Incr. & TNS & Norm. \\
\hline handFP & 38.34 & 1.000 & 2253 & -31.7 & $0 \%$ & -998 & 1.00 \\
\hline IndEDA & \multicolumn{7}{|c|}{ Could not finish routing } \\
\hline HiDaP-M & 38.70 & 1.009 & 2269 & -24.6 & $-4.0 \%$ & -1259 & 1.26 \\
\hline $\mathrm{c5}$ & WL & Norm. & DRC & WNS & Incr. & TNS & Norm. \\
\hline handFP & 38.72 & 1.000 & 518 & -10.0 & $0 \%$ & -48 & 1.00 \\
\hline IndEDA & 44.87 & 1.159 & $>10^{4}$ & -20.5 & $10.53 \%$ & -220 & 4.58 \\
\hline HiDaP-M & 39.12 & 1.014 & 633 & -12.4 & $2.4 \%$ & -29 & 0.58 \\
\hline $\mathrm{c} 6$ & WL & Norm. & DRC & WNS & Incr. & TNS & Norm. \\
\hline handFP & 74.21 & 1.000 & 3169 & -35.8 & $0 \%$ & -1978 & 1.00 \\
\hline IndEDA & 99.14 & 1.336 & $>10^{5}$ & -40.5 & $4.6 \%$ & -389 & 0.20 \\
\hline HiDaP-M & 75.33 & 1.015 & 2869 & -51.0 & $15.2 \%$ & -4409 & 2.23 \\
\hline $\mathrm{c} 7$ & WL & Norm. & DRC & WNS & Incr. & TNS & Norm. \\
\hline handFP & 35.64 & 1.000 & 49 & -32.5 & $0 \%$ & -215 & 1.00 \\
\hline IndEDA & \multicolumn{7}{|c|}{ Could not finish routing } \\
\hline HiDaP-M & 34.28 & 0.962 & 62 & -12.7 & $-19.8 \%$ & -33 & 0.15 \\
\hline $\mathrm{c} 8$ & WL & Norm. & DRC & WNS & Incr. & TNS & Norm. \\
\hline handFP & 24.79 & 1.000 & 5498 & -16.4 & $0 \%$ & -774 & 1.00 \\
\hline IndEDA & 24.85 & 0.987 & 5457 & -34.1 & $17.8 \%$ & -1014 & 1.36 \\
\hline HiDaP-M & 23.95 & 0.951 & 6338 & -16.9 & $0.5 \%$ & -547 & 0.77 \\
\hline
\end{tabular}

all circuits, and the last three over the ones that IndEDA could finish routing (6 circuits of 8 , since it was no able to obtain routing results for $c 4$ and $c 7$ in less than a week). The results show how WL reduction results observed in placement hold after routing, with timing showing an even better reduction $(-4.3 \%$ clock period for WNS, $-70 \%$ TNS) with a similar number of DRC violations. The comparison holds when observing only the circuits IndEDA managed to finish. The results by the industrial tool confirm again the important gap on WL, and although its timing results are better than in the manual designs, the IndEDA layouts have an enormous number of DRC violations.

Detailed results by circuit are shown in Table [VI] The only circuits where the IndEDA macro placements managed to finish with reasonable DRC violations are $c l$ and $c 8$, which are the ones with fewer macros (32, 37 respectively). Our approach HiDaP-M obtained results comparable to handFP in all circuits, specially in $c 2$, where it nearly closed timing, and $c 7$, with a reduction of close to $4 \%$ in WL, $20 \%$ of period in WNS and $85 \%$ of the TNS. On the other hand, HiDaP did not achieve better results in circuits $c 4$ and $c 6$, specially considering timing results.

\section{F. Post-Routing Timing Closure}

In order to check whether the floorplans can serve as initial proposals for industrial block design, a team of engineers took the HiDaP-M layouts at routing stage and tried to close timing without DRC violations. They pushed the macro placements through the full production physical design flow. Four of the 
TABLE VII: Timing-closure results after manual refinement.

\begin{tabular}{|l|r|r|rr|}
\cline { 2 - 5 } \multicolumn{1}{c|}{} & WNS & \multicolumn{1}{c|}{ TNS } & \multicolumn{2}{c|}{ Sign-off? } \\
\hline \multicolumn{1}{c|}{ Case } & \%clk & \multicolumn{1}{c|}{ ns } & DRC & \#FEP \\
\hline$c 5$ & -9.1 & -28.70 & 633 & 2632 \\
$c 5+$ manual & -3.3 & -0.65 & 34 & 112 \\
\hline$c 7$ & -12.5 & -32.84 & 62 & 1528 \\
$c 7+$ manual & -2.5 & -1.54 & 22 & 479 \\
\hline
\end{tabular}

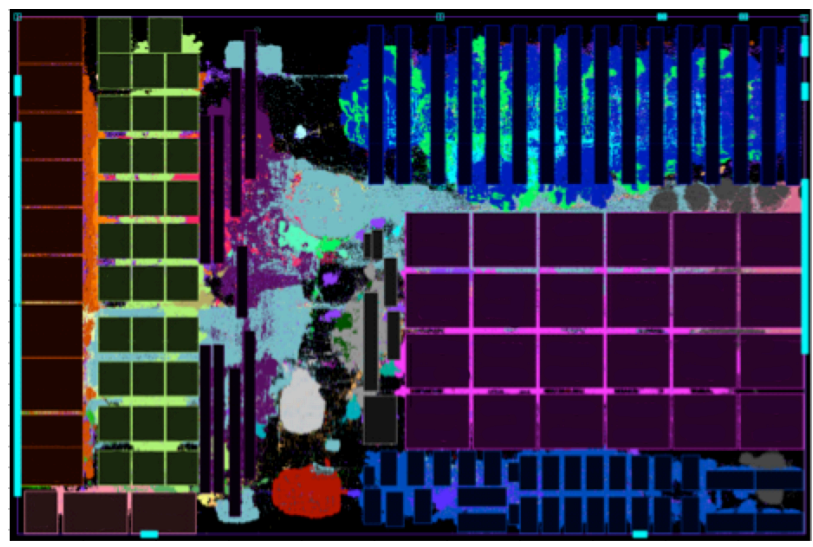

(a) c5 automatic macro placement.

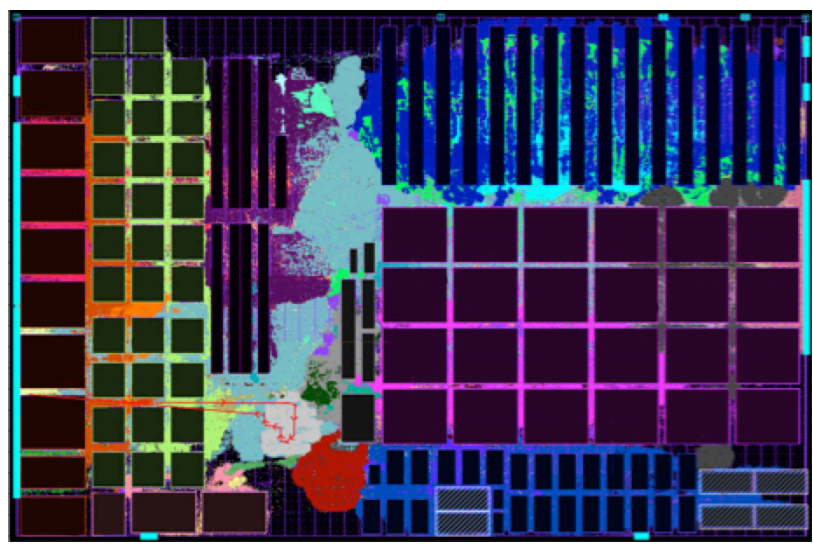

(b) $c 5$ after light manual refinement.

Fig. 19: Layout of $c 5$ after routing.

circuits $(c 1, c 3, c 4, c 7)$ were practically brought to sign-off (residual WNS/TNS and DRC violations) with little macro adjustment (aligning and small moves): that is, at most one iteration of macro refinement. The other circuit were also brought to completion after 2 macro refinement iterations.

Table VII shows detailed results for the process in $c 5$ and $c 7$, with the values for the automated layouts after passing the whole physical design process and after being manually modified by back-end engineers. Columns represent WNS, TNS, number of DRC violations and number of failing endpoints \#FEP. The results after small manual refinement are almost timing closed and DRC clean.

Fig. 19a shows the automatic macro placement generated by HiDaP of the $c 5$ circuit after routing, and Fig. $19 \mathrm{~b}$ after manual modifications, which in this case include rearranging the macros to the left of the central gap, reordering the macros to the top right and adding blockages to the bottom-right. The same kind of modifications have been applied to the layout of $c 7$, as shown in Fig. 1a and Fig. 1b in the introduction.
TABLE VIII: Correlation of Dataflow and Placement Metrics.

\begin{tabular}{|l|rrr|}
\cline { 2 - 4 } \multicolumn{1}{c|}{} & \multicolumn{3}{c|}{ Predicted } \\
\hline Predictor & WL & TNS & WNS \\
\hline Dataflow & 0.156 & 0.075 & 0.045 \\
\hline WL & - & 0.317 & 0.249 \\
\hline TNS & - & - & 0.616 \\
\hline
\end{tabular}

\section{G. Correlation of Dataflow and Placement Metrics}

To understand the correlation between dataflow and circuit quality metrics at the placement stage, the 8 benchmarks were run with 6 different parameter configurations and 5 seeds, generating 240 observations. The dataflow score (of the toplevel floorplan instance, see Fig. 7a, WL, TNS and WNS of each group of 5 runs were normalized against the average in their group, and a linear regression was done to understand the correlation between pairs of predictor and predicted variables.

The results are shown in Table VIII The positive correlations in the first row allow us to infer even small variations in the quality of the dataflow minimization has a positive impact on layout quality: among groups of 5 circuits with optimized dataflow, the ones which achieved lower dataflow yield better results. This is despite the fact that other layout properties such as regularity and channel sizing are not captured by the more global dataflow metric. The other rows provide interesting insight on the relation of WL, TNS and WNS placement results in real FinFET circuits.

\section{CONCLUSIONS}

This paper proposes HiDaP, a tool that exploits RTL information for macro floorplanning using a hierarchical multilevel flow. It performs dataflow analysis to understand data pipelines and the information flow between blocks in the circuit, and uses an adaptive multi-objective cost function aiming at the minimization of wirelength, timing, overlap and distance to preferred macro locations, which can be generated by our proposed analytical methods. The effectiveness of HiDaP has been tested with an industrial suite of benchmarks. Our approach outperforms current industrial and academic state-of-the-art tools and its results are close to those of handcrafted floorplans in WL with generally better timing. Some of the layouts have been brought to almost sign-off with little manual intervention, showing HiDaP is capable of generating promising starting points for engineers to find a final layout in reduced turnaround time.

\section{REFERENCES}

[1] ICCAD 2012 Hierarchy-Aware Placement Contest.

[2] S. N. Adya, S. Chaturvedi, J. A. Roy, D. A. Papa, and I. L. Markov. Unification of Partitioning, Placement and Floorplanning. In ICCAD, pages 550-557, Nov. 2004

[3] S. N. Adya and I. L. Markov. Combinatorial Techniques for Mixed-Size Placement. ACM Trans. Des. Auto. Electr. Syst., 10(1):58-90, 2005.

[4] T. Ajayi, V. A. Chhabria, M. Fogaa, S. Hashemi, A. Hosny, A. B. Kahng, M. Kim, J. Lee, U. Mallappa, M. Neseem, G. Pradipta, S. Reda, M. Saligane, S. S. Sapatnekar, C. Sechen, M. Shalan, W. Swartz, L. Wang, Z. Wang, M. Woo, and B. Xu. Toward an Open-Source Digital Flow: First Learnings from the OpenROAD Project. page 76. ACM, Feb. 2019.

[5] C. C. Hu, D. S. Chen, and Y. W. Wang. Fast Multilevel Floorplanning for Large Scale Modules. In ISCAS, pages 205-208, May 2004.

[6] C. H. Chang, Y. W. Chang, and T. C. Chen. A Novel Damped-Wave Framework for Macro Placement. In ICCAD, pages 504-511, 2017. 
[7] S. T. Chen, Y. W. Chang, and T. C. Chen. An Integrated-SpreadingBased Macro-Refining Algorithm for Large-Scale Mixed-Size Circuit Designs. In ICCAD, pages 496-503, 2017.

[8] T. C. Chen et al. MP-Trees: A Packing-Based Macro Placement Algorithm for Modern Mixed-Size Designs. IEEE Trans. on CAD, 27(9):1621-1634, 2008.

[9] Y. F. Chen et al. Routability-Driven Blockage-Aware Macro Placement. In $D A C$, pages $1-6,2014$

[10] C. Cheng, A. B. Kahng, I. Kang, and L. Wang. RePlAce: Advancing Solution Quality and Routability Validation in Global Placement. IEEE Transactions on Computer-Aided Design of Integrated Circuits and Systems, pages 1-1, 2018.

[11] C. H. Chiou et al. Circular-Contour-Based Obstacle-Aware Macro Placement. In ASP-DAC, pages 172-177, 2016.

[12] W. Choi and K. Bazargan. Hierarchical Global Floorplacement using Simulated Annealing and Network Flow Area Migration. In DATE, pages 1104-1105. IEEE, 2003.

[13] Y. L. Chuang et al. Design-Hierarchy Aware Mixed-Size Placement for Routability Optimization. In ICCAD, pages 663-668, 2010.

[14] J. Cong, M. Romesis, and J. R. Shinnerl. Fast Floorplanning by Look-ahead Enabled Recursive Bipartitioning. IEEE Trans. on CAD, 25(9):1719-1732, Sept. 2006.

[15] T. M. J. Fruchterman and E. M. Reingold. Graph Drawing by ForceDirected Placement. Software Practice \& Experience, 21(11):11291164, Nov. 1991.

[16] M. Hsu, Y. Chen, C. Huang, S. Chou, T. Lin, T. Chen, and Y. Chang. NTUplace4h: A Novel Routability-Driven Placement Algorithm for Hierarchical Mixed-Size Circuit Designs. IEEE Trans. on CAD, 33(12):1914-1927, Dec. 2014.

[17] M. K. Hsu et al. Routability-Driven Placement for Hierarchical MixedSize Circuit Designs. In DAC, pages 1-6, 2013.

[18] D. H. Kim and S. K. Lim. Bus-Aware Microarchitectural Floorplanning. In $A S P-D A C$, pages 204-208. IEEE Computer Society Press, Jan. 2008

[19] M.-C. Kim, N. Viswanathan, C. J. Alpert, I. L. Markov, and S. Ramji. MAPLE: Multilevel Adaptive Placement for Mixed-Size Designs. In ISPD, pages 193-200. ACM, 2012.

[20] Y. Koren. On Spectral Graph Drawing. In Proc. Int. Conf. on Computing and Combinatorics, pages 496-508. Springer-Verlag, July 2003.

[21] J.-M. Lin, Y. Deng, S. Li, B. Yu, L. Chang, and T. Peng. RegularityAware Routability-Driven Macro Placement Methodology for MixedSize Circuits With Obstacles. IEEE Trans. on VLSI Systems, pages $1-12,2018$.

[22] J.-M. Lin, S.-T. Li, and Y.-T. Wang. Routability-Driven Mixed-Size Placement Prototyping Approach Considering Design Hierarchy and Indirect Connectivity Between Macros. In DAC, page 119. ACM, June 2019.

[23] J.-M. Lin, B. H. Yu, and L. Y. Chang. Regularity-Aware RoutabilityDriven Placement Prototyping Algorithm for Hierarchical Mixed-Size Circuits. In ASP-DAC, pages 438-443, Jan. 2017.

[24] Y.-C. Liu, T.-C. Chen, Y.-W. Chang, and S.-Y. Kuo. MDP-trees: MultiDomain Macro Placement for Ultra Large-Scale Mixed-Size Designs. In ASP-DAC, pages 557-562. ACM, Jan. 2019.

[25] V. Nookala, Y. Chen, D. J. Lilja, and S. S. Sapatnekar. MicroarchitectureAware Floorplanning Using a Statistical Design of Experiments Approach. In $D A C$, pages 579-584, June 2005.

[26] R. H. J. M. Otten. Efficient Floorplan Optimization. In ICCD, pages 499-502, 1983.

[27] T. C. Chen and Y. W. Chang. Modern Floorplanning Based on B*-tree and Fast Simulated Annealing. IEEE Trans. on CAD, 25(4):637-650, Apr. 2006.

[28] T. C. Chen, Y. W. Chang, and S. C. Lin. A New Multilevel Framework for Large-Scale Interconnect-Driven Floorplanning. IEEE Trans. on $C A D, 27(2): 286-294$, Feb. 2008

[29] M. Then et al. The More the Merrier: Efficient Multi-Source Graph Traversal. Procs. of the VLDB Endowment, 8(4):449-460, 2014.

[30] A. Vidal-Obiols, J. Cortadella, J. Petit, M. Galceran-Oms, and F. Martorell. RTL-Aware Dataflow-Driven Macro Placement. In DATE, pages 186-191, Mar. 2019.

[31] N. Viswanathan, C. Alpert, C. Sze, Z. Li, and Y. Wei. ICCAD-2012 CAD Contest in Design Hierarchy Aware Routability-driven Placement and Benchmark Suite. In ICCAD, pages 345-348, Nov. 2012.

[32] C. Walshaw. A Multilevel Algorithm for Force-Directed Graph Drawing. In Proceedings of the 8th International Symposium on Graph Drawing, pages 171-182. Springer-Verlag, Sept. 2000.

[33] D. F. Wong and C. L. Liu. A New Algorithm for Floorplan Design. In DAC, pages 101-107, 1986.
[34] J. Z. Yan, N. Viswanathan, and C. Chu. Handling Complexities in Modern Large-Scale Mixed-Size Placement. In DAC, pages 436-441, 2009.

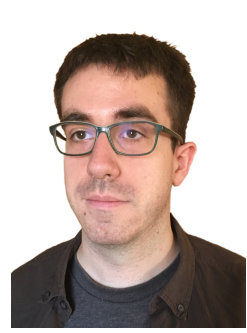

Alex Vidal-Obiols received the M.S. in innovation and research in informatics (2015) and the Ph.D. in computing (2020) from the Universitat Politècnica de Catalunya under the supervision of Prof. Jordi Cortadella and Prof. Jordi Petit. He works as a CAD engineer at Inphi Corporation by expanding and supporting its physical design flows. His research focuses on EDA algorithms for physical design, specially floorplanning, placement and routing in modern industrial flows.

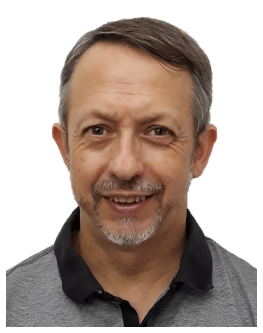

Jordi Cortadella (S'87-M'89-F'15) received the $\mathrm{Ph} . \mathrm{D}$. degree in CS from the Universitat Politècnica de Catalunya, Barcelona, Spain, in 1987. He is a Professor with the Computer Science Department, Universitat Politècnica de Catalunya. His current research interests include formal methods and computer-aided design of VLSI systems with a special emphasis on asynchronous circuits, concurrent systems, and logic synthesis. He received best paper awards at ASYNC 2004 and 2016, DAC 2004, ACSD 2009, and FPGA 2020. He has served on the technical committees of several international conferences in the field of design automation and concurrent systems, and is an Associate Editor of the IEEE Transactions on Computer-Aided Design of Integrated Circuits and Systems.

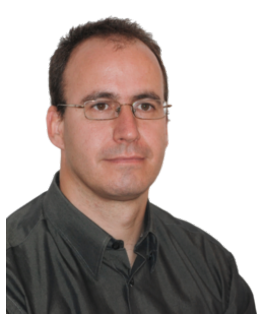

Jordi Petit received the M.S. degree in computing engineering and the Ph.D. degree in computer science from the Universitat Politècnica de Catalunya, Barcelona, Spain, in 1996 and 2001, respectively. Since 2002, he has been an Associate Professor in the Computer Science Department of this university. $\mathrm{He}$ has co-authored numerous research papers published in peer-reviewed journals and international conferences. His current research interests include algorithm engineering. He has worked on topics such as the design, analysis, and implementation of algorithms and data structures (in sequential, distributed, and parallel settings), heuristic approaches to combinatorial optimization problems (e.g., on layout and routing).

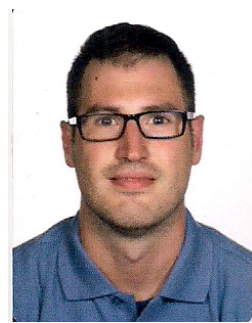

Marc Galceran-Oms Marc Galceran-Oms received the M.S. and Ph.D. degrees in Computer Science from the Universitat Politècnica de Catalunya, Barcelona, in 2007 and 2011. His research interests include formal methods in system design, logic synthesis and EDA algorithms for physical design. $\mathrm{He}$ is currently an ASIC and CAD manager at Inphi. Prior to that he was at an asynchronous design startup Elastix, and as an intern at the Strategic CAD Labs group of Intel.

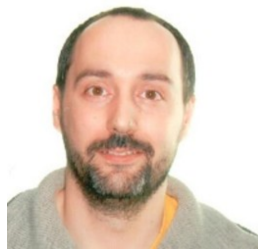

Ferran Martorell Ferran Martorell received the M.S. (2001) and Ph.D. (2009) degrees in electronic engineering from the Universitat Politècnica de Catalunya, Barcelona. Since 2007, he has been working on the ASIC industry developing and leading $\mathrm{R} \& \mathrm{D}$ projects. He has been directly involved on the development of EDA tools, asynchronous circuit methodologies, large ASIC implementations, ASIC methodology from RTL to GDS and bring up. His main research interests include nanotechnology, noise and variability effects and reliability, VLSI design, test, and optimization, computer architecture, computer-aided design, and ASIC design for AI. Currently he is CAD Director at Inphi. 